\title{
Experiencia docente con entornos virtuales en psicología del deporte, antes y durante la pandemia Covid-19
}

\author{
Sergio Barbosa-Granados ${ }^{1}$; Waldino Castañeda-Lozano ${ }^{2}$; Mario Reyes-Bossio*3 \\ ${ }^{1}$ https://orcid.org/0000-0002-2582-2495, Universidad Cooperativa de Colombia, Programa de Psicología, Risaralda, Colombia. \\ 2 https://orcid.org/0000-0003-4267-8154. Universidad Tecnológica de Pereira, Vicerrectoría de Responsabilidad y Bienestar \\ Universitario, Risaralda, Colombia. ${ }^{3}$ https://orcid.org/0000-0003-4655-1927, Universidad Peruana de Ciencias Aplicadas, Facultad \\ de Psicología, Lima, Perú
}

Citar como: Barbosa-Granados, S., Castañeda-Lozano, \& W., Reyes-Bossio, M. (2022). Experiencia docente con entornos virtuales en psicología del deporte, antes y durante la pandemia Covid-19. Revista Digital de Investigación en Docencia Universitaria, 16(1), e1438. https://doi.org/10.19083/ridu.2022.1438

Recibido: 09/02/2021. Revisado: 12/08/2021. Aceptado: 22/10/2021. Publicado: 26/11/2021

\section{Resumen}

Introducción: El objetivo del estudio es presentar una experiencia docente en el campo de la psicología del deporte mediante entornos virtuales en una universidad colombiana, antes y durante la pandemia COVID-19. En tal sentido, se desarrolló el trabajo de intervención psicológica deportiva de evaluación e intervención que, durante la cuarentena, se realizaron en dos etapas, presencial y virtual. Se aplicaron instrumentos psicológicos, con la finalidad de evaluar los niveles de motivación y ansiedad precompetitiva; finalmente se realizaron charlas y conferencias virtuales para deportistas y entrenadores. Dentro de los principales resultados se obtuvo una elevada participación y adherencia a las conferencias y al trabajo de intervención virtual en los estudiantes atletas de la Universidad Tecnológica de Pereira.

Palabras Clave: educación; estudiante universitario; deporte; psicología; tecnología.

\section{Teaching experience with virtual enviroments in sports psychology, before and during the Covid-19 pandemic}

\footnotetext{
Abstract

Introduction: The objective of the study is to present a teaching experience in the field of sport psychology through virtual environments in a Colombian university during the COVID-19 pandemic. In this sense, the work of sports psychological intervention of evaluation and intervention was developed that under the reasons of forty were carried out in two stages, face-to-face and virtual. Psychological instruments were applied in order to evaluate the levels of motivation and precompetitive anxiety; finally, virtual talks and conferences were held for athletes and coaches. Among the main results, high participation and adherence to lectures and virtual intervention work was obtained in student athletes from the Technological University of Pereira.

Keywords: education; college student; sport; psychology; technology.
}

\author{
*Correspondencia: \\ Mario Reyes-Bossio \\ mario.reyes@upc.pe \\ sergio.barbosag@campusucc.edu.co
}




\section{Introducción}

En el marco de la psicología del deporte existe una extensa línea de investigación enfocada en la juventud (Fry et al., 2020) y específicamente en el deporte universitario (Bissett \& Tamminen, 2020; Kucharski et al., 2018; Trujillo \& Reyes-Bossio, 2019). Se evidencia que existen varios factores psicológicos estresantes exclusivos de los estudiantes-atletas, los cuales debemos mirar frente a la carrera dual que desempeñan (Reyes-Bossio, 2020). Todo ello contribuye a experimentar variables negativas en el ámbito psicológico y su relación con las exigencias deportivas en la universidad (e.g, lesiones, presión para lograr el éxito atlético, conflicto interpersonal con compañeros de equipo o entrenadores, demandas de tiempo y horarios, entre otros) (Sudano et al., 2016).

Por consiguiente, los estudiantes-atletas evidencian altas exigencias que repercuten en su bajo bienestar subjetivo, debido al doble rol que cumplen como estudiante y deportista. En consecuencia, los estudiantes universitarios necesitan equilibrar innumerables demandas y desafíos tanto académicos como deportivos (Cho et al., 2020), los cuales se evidencian en la sobrecarga relacionada al tiempo invertido en el entrenamiento, desplazamiento, competición, viajes, entre otros (Brown, 2014).

Adicionalmente, en el primer trimestre del 2020 los deportes en todo el mundo (incluyendo el deporte universitario) dieron un giro inmediato, con cierres inminentes (Gallego et al., 2020; Gautret et al., 2020), debido al riesgo de contagio por parte de los deportistas asociados con la transmisión de enfermedades respiratorias e infecciosas en espacios deportivos (Lin \& Decker, 2010; Parnell et al., 2020). Este contexto ha conllevado a escenarios de estrés, ansiedad, sensibilidad emocional, baja sensación de bienestar y diversas dificultades relacionadas con la salud mental (Henriksen et al., 2019; da Silva et al., 2021; Urzúa et al., 2020); resultado de la situación de confinamiento y de incertidumbre asociada con un mayor efecto de sentimientos negativos (Lades et al., 2020) y sus implicaciones relacionadas con la actividad física (Alarcón \& Hall, 2021).
Sumado a lo anterior, el estudiante-atleta no está exento del riesgo de deserción; sobre este punto, el sistema educativo colombiano, como muchos otros, es aquejado por diversas problemáticas, siendo la deserción estudiantil una de las principales dificultades. Generalmente, los jóvenes universitarios ven como un fracaso el hecho de ingresar al sistema y no llegar a graduarse; esta situación permite observar con claridad el problema que afecta a la educación del país, pues no logra garantizar el egreso y tampoco mantiene a todos los estudiantes que ingresan, limitando de esta manera la cobertura de la educación superior y demorando el capital humano de calidad (Misas, 2004; Ramírez-Gutiérrez et al., 2020).

De ahí que el campo de la psicología del deporte ha prestado especial atención al apoyo del deportista universitario desde el enfoque social, académico y rendimiento deportivo, enfocándolo a un trabajo integral sostenible (Reyes-Bossio, 2020). Conducente en la premisa de que los atletas afronten de mejor manera la situación de confinamiento, basados en objetivos a corto, mediano y largo plazo. Se ha resignificado su trayectoria deportiva y dando sentido a sus posibles resultados en el futuro (Schinke et al., 2020).

De este modo, este artículo tiene como objetivo presentar una experiencia docente en el campo de la psicología del deporte, mediante entornos virtuales, en una universidad colombiana durante la pandemia COVID-19.

\section{Marco Teórico}

\section{Descripción de la entidad deportiva}

La Universidad Tecnológica de Pereira (UTP) es una institución de educación superior que se encuentra ubicada en la ciudad de Pereira (Colombia), la cual cuenta con una población de 18.000 estudiantes aproximadamente, 10 facultades y 35 programas de pregrado. Adicionalmente, la UTP es una institución acreditada como una entidad de expresión cultural y patrimonio de la región, de carácter oficial seccional.

En este contexto, el procedimiento deportivo de la Universidad Tecnológica de Pereira se encuentra regido bajo normativa de la Asociación 
Colombiana de Universidades (ASCUN) deportes. El proceso formativo en el aspecto deportivo es fundamental para la UTP debido a que complementa la formación académica, así como la búsqueda de valores agregados como: disciplina, responsabilidad, compañerismo, solidaridad, compromiso, entre otros. Así mismo, se tiene en cuenta los múltiples beneficios que genera la actividad física, resaltando el beneficio físico y mental de los individuos. Proporcionando el equilibrio de su propio bienestar, la capacidad de superación, retos y liderazgo.

Por tanto, el deporte competitivo en la UTP cuenta actualmente con 22 equipos deportivos. Los seleccionados deportivos participan en el ciclo competitivo de la ASCUN, los cuales se componen de una primera fase denominada Juegos Zonales Universitarios en los que participan los departamentos (estados) del Huila, Caquetá, Cundinamarca, Choco, Risaralda, Quindío, Tolima y Caldas. Después de este clasificatorio los mejores resultados pasan a la segunda fase denominada Juegos Nacionales Universitarios, en los que compiten los mejores deportistas del país. En la última versión de estos juegos llevada a cabo en la ciudad de Barranquilla, en el mes de septiembre del 2019, la UTP contó con la participación de 128 deportistas pertenecientes a 14 modalidades deportivas consiguiendo resultados positivos. Adicionalmente, en el ciclo internacional los deportistas de la UTP han participado históricamente en Sudamericanos, Centroamericanos, Panamericanos, Mundiales y Universiadas.

\section{Descripción de actividades}

La presente experiencia de trabajo se realizó durante una etapa previa al confinamiento y durante el confinamiento por la COVID-19 (World Health Organization, 2020). La Universidad Tecnológica de Pereira y sus seleccionados deportivos tuvieron que adaptarse a este cambio y nueva normalidad (Liu et al., 2020; Shultz et al., 2020; Y. Yang et al., 2020). En tal sentido, la presente experiencia de trabajo sufrió algunos cambios en su planificación inicial y se adaptó para cumplir con las necesidades institucionales y el cumplimiento de aislamiento social. Las tareas realizadas fueron divididas en tres momentos: (i) preparación, (ii) observación e (iii) intervención. A continuación, se presentan de manera detallada.

\section{Preparación}

En el periodo de preparación (etapa sin confinamiento) que comprendió las primeras tres semanas. En este periodo, se adelantaron reuniones con el equipo de deportes de la vicerrectoría de bienestar universitario, incluyendo los entrenadores de los diferentes selectivos de la UTP. A partir de estas reuniones iniciales, se identificó de primera mano las dificultades de los selectivos y los casos particulares de atletas, permitiendo de esta manera, el análisis y diagnóstico de necesidades de los deportistas UTP. Dentro de las necesidades que se manifestaron en este diagnóstico, se evidencia la variable ansiedad precompetitiva (Arenas-Granada et al., 2016; Pons et al., 2016; H. Yang et al., 2020) como variable más importante para trabajar con los selectivos deportivos y con los casos particulares se resalta la falta de motivación (Garcia-Mas et al., 2011; Starzak \& Sas-Nowosielski, 2019) y la necesidad de establecimiento de metas y objetivos (Castro-Sánchez et al., 2018; Reyes-Bossio, 2009). Es por lo anterior, que se diseña el plan de trabajo encaminado con el objetivo de atender temas relacionados con estas variables en los seleccionados de taekwondo y atletismo.

\section{Observación}

La observación históricamente ha sido una metodología utilizada para el estudio del comportamiento habitual de los deportistas. Establecido en el uso de la percepción visual como un elemento clave del comportamiento, permite registrar los estados emocionales del deportista o del entrenador. Configurándose como una nueva estructura flexible que considera el procedimiento científico a partir de datos de la realidad perceptible con posibles análisis ulteriores (Anguera \& Hernández-Mendo, 2014).

En consecuencia, esta etapa observacional tuvo dos momentos, la primera se realizó antes de la cuarentena, y la segunda al inicio de la cuarentena. La fase de observación empezó con la presentación del psicólogo deportivo en diferentes espacios de reunión e introducción con los deportistas de los seleccionados de taekwondo y 
atletismo. En este contexto, se aprovecharon dos semanas para asistir a los entrenamientos con el objetivo de observar a los deportistas y su interacción en el campo de juego (Martin et al., 2017; Torres-Ramírez et al., 2014). No obstante, y a partir de lo anteriormente mencionado frente a la situación de confinamiento se desarrolló una segunda etapa de observación indirecta (Bo et al., 2020; Di Fronso et al., 2020; Schinke, Papaioannou, Henriksen et al., 2020) donde se realizaron reuniones de planeación y observación con entrenadores con el objetivo de realizar actividades que permitieran apoyar a los estudiantes deportistas o a la comunidad universitaria en temas relacionados con la psicología del deporte en la etapa de pandemia.

\section{Intervención}

El proceso de intervención también tiene dos etapas marcadas, el antes del confinamiento y el durante el confinamiento. La intervención antes del confinamiento se inicia con el trabajo de establecimiento de metas y objetivos con cada uno de los deportistas seleccionados de atletismo y taekwondo, con la finalidad de conocer su proyección para el presente año en el aspecto competitivo. Se tiene como evento más importante la preparación para los Juegos Zonales Universitarios a realizarse en abril y mayo del 2020 (los cuales fueron cancelados por la COVID en el mes de marzo del 2020). Para esto, se realiza una serie de entrevistas a los deportistas con la intención de conocer sus metas para la etapa competitiva, evidenciando que la mayoría de los atletas-estudiantes están enfocados en la clasificación y posterior logro de medallas en los Juegos Nacionales Universitarios. En este sentido, la entrevista recoge el máximo de información posible relacionado con diferentes áreas de la vida deportiva, resaltando su historia deportiva, recursos económicos, relaciones personales, organización del tiempo, descanso, alimentación, lesiones previas, resultados deportivos, objetivos, relaciones con compañeros de equipo, entrenamiento y competición (González, 2010).

Posteriormente se aplicaron pruebas en el campo de la psicología del deporte para medir niveles de ansiedad precompetitiva y motivación en los deportistas de la UTP; entendiendo la ansiedad precompetitiva como una reacción emocional de aprehensión y tensión ante situaciones de competencia deportiva, en las cuales se evalúan o miden un conjunto de elementos combinados de conocimientos, habilidades, actitudes y saberes en el campo deportivo (Olmedilla et al., 2019; Ong \& Chua, 2021). Paralelamente, la motivación en el deporte es entendida como un elemento clave para lograr el compromiso y la adherencia al deporte, ya que es el más importante e inmediato determinante del comportamiento humano y se encuentra mediado por la dirección e intensidad del propio esfuerzo (Aguirre-Loaiza \& González, 2014; Weinberg \& Gould, 2010).

Las pruebas que se utilizaron fueron las siguientes: Inventario de Ansiedad Estado Competitiva -2 Revisado (CSAI-2R), que mide tres factores la ansiedad somática, ansiedad cognitiva y autoconfianza. Adicionalmente, se utilizó la Escala de Motivación Deportiva (EMD), que mide tres factores: motivación intrínseca, motivación extrínseca y amotivación. Estas pruebas permitieron recoger información relevante que ayudaría para la implementación de las sesiones virtuales.

Así mismo, después de la aplicación de las pruebas y su posterior análisis, se evidenció que la autoconfianza es la variable que mejor puntúa para los deportistas de atletismo y taekwondo. En lo que respecta a la ansiedad somática el selectivo de taekwondo puntuó más alto y en contraste frente la ansiedad cognitiva el selectivo de atletismo puntuó en mayor medida. Por su parte, en lo que respecta a la variable motivación; se determina que el selectivo de atletismo está más relacionado con la motivación intrínseca (e.g, diversión, placer y satisfacción por el deporte, mejora de las tareas deportivas). Sin embargo, el selectivo de taekwondo está más relacionado con la motivación extrínseca (e.g, reconocimiento, trofeos, beneficios económicos, becas académicas, refuerzos ambientales).

No obstante que los datos permitirían conocer la situación de los deportistas en lo referente a las variables evaluadas, lastimosamente en el momento en que se realizarían las intervenciones basadas en los protocolos realizados por Trujillo y Reyes-Bossio (2019), enfocados al trabajo relacio- 
nado con el manejo de la ansiedad y motivación, la UTP por requerimientos nacionales determinó el cierre de la universidad incluyendo cualquier actividad deportiva dentro de los espacios de la institución educativa.

En este contexto, se da la segunda etapa de intervención y el equipo de deportes UTP toma la decisión de continuar las actividades con los diferentes selectivos de manera virtual (Dijkstra et al., 2020), durante el resto del semestre y solicitando al psicólogo deportivo acompañar actividades encaminadas a la comunidad universitaria deportiva. Es por lo anterior que, desde la práctica, se comienza a realizar una serie de conferencias sobre temáticas relacionadas con la psicología deportiva para la comunidad UTP, contando con el apoyo y promoción de actividades conjuntas con el Colegio Colombiano de Psicólogos (COLPSIC), la Universidad Cooperativa de Colombia (UCC) y el programa en Ciencias del Deporte y la Recreación de la UTP (ver tabla 1).

En consecuencia, se desarrolla una encuesta virtual (Google Forms) con el objetivo de determinar el impacto de las actividades realizadas en la comunidad deportiva de la Universidad Tecnológica de Pereira y los niveles de satisfacción frente a las conferencias. Los resultados de la encuesta evidencian que la mayoría de los par- ticipantes son estudiantes universitarios que forman parte de los diferentes selectivos de la UTP. No obstante, se determina que sería muy probable recomendar este tipo de actividades virtuales a otros deportistas o colegas. En este sentido, el nivel de satisfacción que tienen los participantes frente a la conferencia es significativo, pero se debe mejorar el tiempo asignado para las discusiones concerniente a la respuesta de inquietudes de los participantes. Adicionalmente, se resalta el uso de WhatsApp como herramienta líder para la comunicación relacionada con la convocatoria a este tipo de conferencias y su afinidad con eventos deportivos (Ahmed et al., 2020; Tamir, 2020).

En este sentido y teniendo en cuenta cuál es el objetivo principal para asistir a este tipo de conferencia, los encuestados resaltan que el adquirir herramientas de la psicología del deporte es el más importante, seguido del objetivo de aprender sobre psicología del deporte y descubrir contenido sobre la psicología del deporte (Raabe et al., 2019).

Por último, y en cuanto a lo cualitativo, los participantes frente a la pregunta ¿Qué fue lo que más te gustó de las conferencias sobre psicología del deporte?, respondieron: "el contenido en general fue importante", "el contenido temático que se le dio a las conferencias, explicando sobre la importancia en estos momentos de pandemia

\section{Tabla 1}

Conferencias sobre psicología deportiva durante cuarentena

Título de la conferencia

Fecha

La información en tiempos de crisis: Aportes desde algunos campos de la psicología. (COLPSIC)

Psicología del deporte una mirada a la cognición y el rendimiento deportivo. (UCC) 
y cómo volver a la práctica progresivamente". Finalmente, frente a la pregunta ¿qué temas te gustaría que se trataran en conferencias futuras?, los encuestados respondieron "estrategias que puedan mejorar la concentración y la ansiedad antes de una competencia" y "dar un número mayor de herramientas para aplicar en situación de pandemia y después de la pandemia".

A partir de las conferencias y entendiendo que las redes sociales son un espacio cada vez mayor para la comunicación interpersonal y masiva (Dietrich et al., 2012; Romney \& Johnson, 2020), se crea la página de Instagram: @psicodeporteu,con el propósito de estar más cerca de la comunidad universitaria y especialmente con los diferentes selectivos deportivos de la UTP. Lo anterior con el objetivo de interactuar, subir información relevante desde la psicología del deporte. La cual, permite generar un acompañamiento en el periodo de confinamiento desde las nuevas tecnologías. Se entiende también de esta forma la capacidad de innovar y llegar a la población objeto que en su gran mayoría son adolescentes y jóvenes adultos (García del Castillo et al., 2020; Papalia et al., 2012).

Así mismo, se mantiene asistencia constante a las reuniones con entrenadores para conocer casos específicos de los deportistas de la UTP. Estas reuniones permitieron conocer las estrategias que vienen trabajando los entrenadores con sus deportistas y se identifican las necesidades de intervención para el apoyo desde el aspecto de la psicología del deporte (Aguirre-Loaiza et al., 2020; Stambulova et al., 2020; Taku \& Arai, 2020) y a partir de estos insumos nacen las conferencias y reuniones anteriormente presentadas (ver Tabla 1).

\section{Discusión}

El principal objetivo del estudio es presentar una experiencia de trabajo en el campo de la psicología del deporte en ambientes universitarios, antes y durante la pandemia COVID-19, y así establecer líneas de trabajo que permitan potencializar los procesos en el campo de la psicología del deporte en ambientes universitarios virtuales.

La experiencia recogida desde la práctica profesional del psicólogo deportivo en estos contextos de pandemia ha llevado a un proceso de adaptación sostenida por parte de estudiantes, deportistas, docentes y profesionales de las ciencias del deporte. Es así que los procesos virtuales como el teletrabajo, la videoconferencia, el tele estudio, las prácticas deportivas a través del internet, entre otras modalidades virtuales, han tomado una forma de vida dentro del contexto actual para muchos deportistas y estudiantes que deben ir conociendo cada vez más e integrando a sus esquemas de trabajo deportivo y académico (Aquilina \& Henry, 2010; Feito, 2020; Reyes-Bossio, 2021).

Esta interacción, a partir de herramientas virtuales se ha convertido en una habilidad que actualmente transversaliza las estructuras académicas de manera significativa, generando un desarrollo exponencial del conocimiento (Barbosa-Granados \& Amariles, 2019). Las cuales apoyan los procesos educativos y siendo altamente motivantes para los estudiantes-atletas. Por tanto, la labor del psicólogo del deporte estará muy relacionada con las nuevas tecnologías y así mismo, permitirá ofrecer programas encaminados a la capacitación individualizada y grupal de los deportistas (Weinberg et al., 2012). Por ello, esta experiencia de trabajo se ha podido integrar a las competencias académicas, profesionales, deportivas y sociales para que los estudiantes-atletas puedan seguir enfocados en sus objetivos deportivo-académicos y no pierdan el enfoque inicial del cumplimiento de sus objetivos.

Por otro lado, es de resaltar que la base ética y deontológica de la práctica de la psicología del deporte (Colegio Colombiano de Psicólogos, 2016; Etzel \& Skvarla, 2017; Quartiroli et al., 2020) es clave en esta etapa de confinamiento. En tal sentido, el uso adecuado de la telepsicología (Perrin et al., 2020) se encuentra al servicio de los deportistas (Reid et al., 2015) para su bienestar integral. Sin embargo, el progreso tecnológico también ha tenido consecuencias y riesgos para la privacidad del usuario. Por ende, se debe propender por la seguridad y privacidad de datos de los deportistas con los que se trabaja, debido a que, sin privacidad y confidencialidad, el trabajo realizado con los usuarios (estudiantes-atletas) puede no ser eficaz (Lustgarten et al., 2020; Stoll et al., 2020).

Lo anterior, permitirá replantear la labor del psicólogo del deporte dentro del ambiente compe- 
titivo en situación de pandemia y pospandemia, aportando sus conocimientos en la actuación de los deportistas frente a los protocolos de salud, manejo del aislamiento social, retiro de la carrera deportiva, motivación, autocuidado, salud física y salud mental (Barbosa-Granados \& Urrea, 2018), encaminado a la constante búsqueda de la excelencia deportiva y académica (Schinke et al., 2020).

Adicionalmente, a largo plazo los investigadores y equipos universitarios deben abordar la salud mental por la incertidumbre que genera a largo plazo en los estudiantes-atletas la "nueva normalidad", incluyendo un enfoque específico relacionado con lo financiero, competitivo, educativo y social (Håkansson et al., 2020). En este sentido, se debe trabajar en la identidad atlética de los deportistas universitarios, debido al periodo de confinamiento que aún se mantiene en Colombia y que ha afectado la temporada de competencias universitarias. Lo anterior, se puede reforzar con estrategias de afrontamiento y manejo de aspectos emocionales (Costa et al., 2020).

Ahora bien, en el momento de presentar las experiencias realizadas, es necesario considerar las propias limitaciones, las cuales fueron variadas, debido a que no existía experiencia previa relacionada con el trabajo durante periodos de confinamiento. Convirtiéndose lo anterior en un gran reto dentro del trabajo interdisciplinario con los diferentes selectivos de la UTP, desde el trabajo de campo de la psicología del deporte. Sin embargo, la capacidad de adaptación y el interés por parte de la coordinación de deportes de la Vicerrectoría de Responsabilidad Social y Bienestar Universitario (VRSBU) de la UTP permitieron que esta experiencia de trabajo se pudiera llevar a cabo y de esta forma, generar impacto dentro de la comunidad universitaria. Se logró el acceso a los deportistas universitarios, el apoyo de los entrenadores y la apropiación de los espacios asignados.

Agradecimientos: Agradecemos a la Vicerrectoría de Responsabilidad Social y Bienestar Universitario, estudiantes-atletas y profesores del área de deportes de la Universidad Tecnológica de Pereira. Así como también, a los coordinadores y docentes del máster de la Sociedad Iberoamericana de Psicología del Deporte.

\section{Referencias}

Aguirre-Loaiza, H., Arenas-Granada, J.A., Barbosa-Granados, S., \& Herrera-Agudelo, L. (2020). Psicología de la actividad física y el deporte: tendencias, retos y oportunidades antes y después del COVID-19. En N. Molina (Ed.), Psicología en contextos de COVID-19, desafíos poscuarentena en Colombia (pp. 115-127). Asociación Colombiana de Facultades de Psicología. https://www.ascofapsi.org.co/index.php/ blog/blog-with-right-sidebar/246-psicologia-encontextos-de-covid-19

Aguirre-Loaiza, H., \& González, J. (2014). Factores motivacionales, variables deportivas y sociodemográficas en deportistas universitarios. Tesis Psicológica, 9(1), 130-145. https://www.redalyc.org/ pdf/1390/139031679011.pdf

Ahmed, O., Carmody, S., Walker, L. J., \& Ahmad, I. (2020). The need for speed! 10 ways that WhatsApp and instant messaging can enhance communication (and clinical care) in sport and exercise medicine. British Journal of Sports Medicine, 54(19), 1128-1129 . https://doi.org/10.1136/bjsports-2019-101707

Alarcón, E. I., \& Hall, J. A. (2021). Actividad física en estudiantes deportistas universitarios, previo y en el confinamiento por pandemia asociada al COVID-19. Retos, 39, 572-575. https://recyt.fecyt.es/index.php/ retos/article/view/81293/61256

Anguera, M. T., \& Hernández-Mendo, A. (2014). Metodología observacional y psicología del deporte: Estado de la cuestión. Revista de Psicología Del Deporte, 23(1), 103-109. https://www.redalyc.org/ pdf/2351/235129571018.pdf

Aquilina, D., \& Henry, I. (2010). Elite athletes and university education in Europe: A review of policy and practice in higher education in the European Union Member States. International Journal of Sport Policy, 2(1), 25-47. https://doi.org/10.1080/19406941003634024

Arenas-Granada, J. A., Castellanos, V., Aguirre-Loaiza, H., Trujillo, C., \& Núñez, C. A. (2016). La ansiedad en voleibolistas universitarios: análisis de la competencia deportiva en la educación superior. Revista Científica Guillermo de Ockham, 14(2), 1-19. https:// www.redalyc.org/articulo.oa?id=105346890002

Balaguer, I., Castillo, I., \& Duda, J. (2007). Propiedades psicométricas de la escala de motivación deportiva en deportistas españoles. Revista Mexicana de Psicología, 24(2), 197-207. http://www.redalyc.org/articulo.oa?id=243020637005

Barbosa-Granados, S. H., \& Amariles, M. (2019). Learning styles and the use of ICT in university students within a competency-based training model. Journal of New Approaches in Educational Research, 8(1), 1-6. https://dialnet.unirioja.es/servlet/articulo? codigo $=6369972$

Barbosa-Granados, S., \& Urrea, A. (2018). Influencia del 
deporte y la actividad física en el estado de salud físico y mental. Katharsis, 25, 141-160. https://doi. org/10.25057/25005731.1023

Bissett, J., \& Tamminen, K. (2020). Student-athlete disclosures of psychological distress: Exploring the experiences of university coaches and athletes. Journal of Applied Sport Psychology, 1-21. https://doi.org/1 $0.1080 / 10413200.2020 .1753263$

Bo, H. X., Li, W., Yang, Y., Wang, Y., Zhang, Q., Cheung, T., Wu, X., \& Xiang, Y. T. (2020). Posttraumatic stress symptoms and attitude toward crisis mental health services among clinically stable patients with COVID-19 in China. Psychological Medicine, 51(6), 1052 - 1053. https://doi.org/10.1017/ S0033291720000999

Brown, G. T. (2014). Mind, Body and Sport: Understanding and supporting student-athlete mental wellness. National Collegiate Athletic Association. http:// www.ncaa.org/sport-science-institute/mind-bodyand-sport-understanding-and-supporting-student-athlete-mental-wellness

Castro-Sánchez, M., Zurita, F., Chacón, R., López-Gutiérrez, C., \& Zafra, E. O. (2018). Emotional intelligence, motivational climate and levels of anxiety in athletes from different categories of sports: Analysis through structural equations. International Journal of Environmental Research and Public Health, 15(5). https://doi.org/10.3390/ijerph15050894

Cho, H., Yi Tan, H., \& Lee, E. (2020). Importance of perceived teammate support as a predictor of student-athletes' positive emotions and subjective well-being. International Journal of Sports Science \& Coaching, 15(3), 364-374. https://doi. org/10.1177/1747954120919720

Colegio Colombiano de Psicólogos. (2016). Deontología y bioética del ejercicio de la psicología en Colombia. Manual Moderno.

Costa, S., Santi, G., Di Fronso, S., Montesano, C., Di Gruttola, F., Ciofi, E. G., Morgilli, L., \& Bertollo, M. (2020). Athletes and adversities: athletic identity and emotional regulation in time of COVID-19. Sport Sciences for Health, 16(4), 609-618. https://doi. org/10.1007/s11332-020-00677-9

Di Fronso, S., Costa, S., Montesano, C., Di Gruttola, F., Ciofi, E. G., Morgilli, L., Robazza, C., \& Bertollo, M. (2020). The effects of COVID-19 pandemic on perceived stress and psychobiosocial states in Italian athletes. International Journal of Sport and Exercise Psychology, 1-13. https://doi.org/10.1080/161219 7X.2020.1802612

Dietrich, F., Shipherd, A. M., Gershgoren, L., Filho, E. M., \& Basevitch, I. (2012). Sport psychology group consultation using social networking web sites. Psychological Services, 9(3), 323-324. https://doi. org/10.1037/a0026114

Dijkstra, H. P., Ergen, E., Holtzhausen, L., Beasley, I., Alonso, J. M., Geertsema, L., Geertsema, C., Nelis, S., Ngai,
A., Stankovic, I., Targett, S., \& Andersen, T. E. (2020). Remote assessment in sport and exercise medicine (SEM): a narrative review and teleSEM solutions for and beyond the COVID-19 pandemic. British Journal of Sports Medicine 54(19), 1-7. https://doi. org/10.1136/bjsports-2020-102650

Etzel, E. F., \& Skvarla, L. A. (2017). Ethical Considerations in Sport and Performance Psychology. Oxford Research Encyclopedia of Psychology. https://doi. org/10.1093/acrefore/9780190236557.013.141

Feito, R. (2020). Este es el fin de la escuela tal y como la conocemos. Unas reflexiones en tiempo de confinamiento. Revista de Sociología de La Educación-RASE, 13(2), 156-163. https://doi.org/10.7203/ rase.13.2.17130

Fry, M., Reid-Pinson, C., Iwasaki, S., \& Thompson, J. (2020). Bridging Theory, Research, and Practice in Youth Sports: Sport Psychology's Partnership with Positive Coaching Alliance to Enhance Youth Sport. Journal of Sport Psychology in Action, 11(1), 6-19. https://doi.org/10.1080/21520704.2019.1649336

Gallego, V., Nishiura, H., Sah, R., \& Rodriguez-Morales, A. J. (2020). The COVID-19 outbreak and implications for the Tokyo 2020 Summer Olympic Games. Travel Medicine and Infectious Disease, 34. https://doi. org/10.1016/j.tmaid.2020.101604

Garcia-Mas, A., Palou, P., Smith, R. E., Ponseti, X., Almeida, P., Lameiras, J., Jiménez, R., \& Leiva, A. (2011). Performance anxiety and motivational climate in young competitive soccer players in relation to performance and skills perceived by their coaches / Ansiedad competitiva y clima motivacional en jóvenes futbolistas de competición, en relación con las habili. Revista de Psicologia Del Deporte, 20(1), 197-207.

García del Castillo, J. A., García del Castillo-López, Á., Dias, P. C., \& García-Castillo, F. (2020). Social networks as tools for the prevention and promotion of health among youth. Psicologia: Reflexao e Critica, 33, 13. https://doi.org/10.1186/s41155-020-00150-z

Gautret, P., Al-Tawfiq, J., \& Hoang, V. T. (2020). COVID 19: Will the 2020 Hajj pilgrimage and Tokyo Olympic Games be cancelled? Travel Medicine and Infectious Disease, 34. https://doi.org/10.1016/j. tmaid.2020.101622

González, M. D. (2010). Evaluación psicológica en el deporte: aspectos metodológicos y prácticos. Papeles Del Psicólogo, 31(3), 250-258.

Håkansson, A., Moesch, K., Jönsson, C., \& Kenttä, G. (2020). Potentially Prolonged Psychological Distress from Postponed Olympic and Paralympic Games during COVID-19-Career Uncertainty in Elite Athletes. International Journal of Environmental Research and Public Health, 18(1), 2. https://doi.org/10.3390/ ijerph18010002

Henriksen, K., Schinke, R. J., Moesch, K., McCann, S., Parham, W. D., Larsen, C. H., \& Terry, P. C. (2019). Con- 
sensus statement on improving the mental health of high performance athletes. International Journal of Sport and Exercise Psychology, 18(5) 1-8. https://doi.org/10.1080/1612197x.2019.1570473

Kucharski, B., Strating, M. A., Ahluwalia, A., \& Pascual-Leone, A. (2018). Complexity of emotion regulation strategies in changing contexts: A study of varsity athletes. Journal of Contextual Behavioral Science, 10, 85-91. https://doi.org/10.1016/j.jcbs.2018.09.002

Lades, L., Laffan, K., Daly, M., \& Delaney, L. (2020). Daily emotional well-being during the COVID-19 pandemic. British Journal of Health Psychology, 25, 902-911. https://doi.org/10.1111/bjhp.12450

Lin, L., \& Decker, C. F. (2010). Respiratory Tract Infections in Athletes. Disease-a-Month, 56(7), 407-413. https:// doi.org/10.1016/j.disamonth.2010.05.001

Liu, S., Yang, L., Zhang, C., Xiang, Y. T., Liu, Z., Hu, S., \& Zhang, B. (2020). Online mental health services in China during the COVID-19 outbreak. The Lancet Psychiatry, 7(4), e17-e18. https://doi.org/10.1016/ S2215-0366(20)30077-8

Lustgarten, S. D., Garrison, Y. L., Sinnard, M. T., \& Flynn, A.W.P. (2020). Digital privacy in mental healthcare: current issues and recommendations for technology use. Current Opinion in Psychology, 36, 25-31. https://doi.org/10.1016/j.copsyc.2020.03.012

Martin, E. A., Winter, S., \& Holder, T. (2017). An exploration of trainee practitioners' experiences when using observation. Sport Psychologist, 31(2), 160-172. https://doi.org/10.1123/tsp.2016-0019

Misas, G. (2004). La educación superior en Colombia: análisis y estrategias para su desarrollo. Universidad Nacional de Colombia.

Olmedilla, A., Ruiz-Barquín, R., Ponseti, F. J., Robles-Palazón, F. J., \& García-Mas, A. (2019). Competitive Psychological Disposition and Perception of Performance in Young Female Soccer Players. Frontiers in Psychology, 10, 1168. https://doi.org/10.3389/ fpsyg.2019.01168

Ong, N. C. H., \& Chua, J. H. E. (2021). Effects of psychological interventions on competitive anxiety in sport: A meta-analysis. Psychology of Sport and Exercise, 52, 101836. https://doi.org/10.1016/j. psychsport.2020.101836

Papalia, D. E., Duskin, R., \& Martorell, G. (2012). Desarrollo Humano (12th ed.). Mc Graw-Hill.

Parnell, D., Widdop, P., Bond, A., \& Wilson, R. (2020). COVID-19, networks and sport. Managing Sport and Leisure, 1-7. https://doi.org/10.1080/23750472.2020. 1750100

Perrin, P. B., Rybarczyk, B. D., Pierce, B. S., Jones, H. A., Shaffer, C., \& Islam, L. (2020). Rapid telepsychology deployment during the COVID 19 pandemic: A special issue commentary and lessons from primary care psychology training. Journal of Clinical Psychology, 76(6), 1173-1185. https://doi.org/10.1002/ jclp.22969
Pons, J., Ramis, Y., Garcia, A., López de la Llave, A., \& Pérez-Llantada, M. C. (2016). Percepción de la ansiedad competitiva en relación al nivel de cooperación y compromiso deportivo en jugadores de baloncesto de form. Cuadernos de Psicologia Del Deporte, 16(3), 45-54.

Quartiroli, A., Harris, B. S., Brückner, S., Chow, G. M., Connole, I. J., Cropley, B., Fogaça, J., Gonzalez, S. P., Guicciardi, M., Hau, A., Kao, S., Kavanagh, E. J., Keegan, R. J., Li, H. Y., Martin, G., Moyle, G. M., Noce, F., Peterson, K., Roy, J., ... Zito, M. (2020). The International Society of Sport Psychology Registry (ISSP-R) ethical code for sport psychology practice. International Journal of Sport and Exercise Psychology, 1-22. https://doi.org/10.1080/1612197X.2020.1789317

Raabe, J., Bejar, M., Bass, A. D., Lauer, E. E., Erdner, S. M., \& Zakrajsek, R. (2019). Teach Me and I May Remember, Involve Me and I Learn: Four Approaches to Peer Mentoring in Sport Psychology. Quest, 71(4), 447462. https://doi.org/10.1080/00336297.2018.1544082

Ramírez-Gutiérrez, Z., Barrachina, M., \& Ripoll, V. (2020). Eficiencia en la educación superior. Estudio empírico en universidades públicas de Colombia y España. Revista de Administracao Publica, 54(3), 468-500. https://doi.org/10.1590/0034-761220190232

Reid, C., Campbell, C., Locke, V., \& Charlesworth, R. (2015). Australian Men's Hockey Team: Virtually There. Telepsychology in Olympic Sport. Australian Psychologist, 50(4), 279-284. https://doi.org/10.1111/ ap.12138

Reyes-Bossio, M. (2009). Clima motivacional y orientacion de meta en futbolistas peruanos de primera división. Cuadernos De Psicología Del Deporte, 9(1), 5-20. https://revistas.um.es/cpd/article/view/75341

Reyes-Bossio, M. (2020). El deporte Universitario: Asesoramiento, abordaje integral e intervención psicológica deportiva. En J. Trujillo (Ed.), Teoría y práctica de la Psicología del Deporte en Iberoamérica (pp. 240-261). Sociedad Iberoamericana de Psicología del Deporte.

Reyes - Bossio, M. (2021). La academia (escuela - universidad) y el deporte ¿Cómo llevarlo psicológicamente activo? En R. Brandão, J. Dosil, A. Viñolas y R. Domínguez (Eds.), Píldoras Solidarias. Estrategias psicológicas en el deporte para afrontar momentos de crisis (págs. 229-236). Xunta de Galicia y Sociedad Iberoamericana de Psicología del Deporte. chrome-extension://efaidnbmnnnibpcajpcglclefindmkaj/ viewer.html?pdfurl=http\%3A\%2F\%2Fwww.sipd. org\%2Fwp-content\%2Fuploads\%2F2021\%2F10\%2FXuntaDeportes-PildorasES-Completo-web.pdf\&clen $=2570209 \&$ chunk $=$ true

Romney, M., \& Johnson, R. G. (2020). Show me a story: narrative, image, and audience engagement on sports network Instagram accounts. Information, Communication \& Society, 23(1), 94-109. https://doi.org /10.1080/1369118X.2018.1486868 
Schinke, R., Papaioannou, A., Henriksen, K., Si, G., Zhang, L., \& Haberl, P. (2020). Sport psychology services to high performance athletes during COVID-19. International Journal of Sport and Exercise Psychology, 18(3), 1-4. https://doi.org/10.1080/161219 7X.2020.1754616

Schinke, R., Papaioannou, A., Maher, C., Parham, W. D., Larsen, C. H., Gordin, R., \& Cotterill, S. (2020). Sport psychology services to professional athletes: working through COVID-19. International Journal of Sport and Exercise Psychology, 18(4), 409-413. https:// doi.org/10.1080/1612197X.2020.1766182

Shultz, J., Perlin, A., Saltzman, R. G., Espinel, Z., \& Galea, S. (2020). Pandemic March: COVID-19's first wave circumnavigates the globe. Disaster Medicine and Public Health Preparedness, 14(5), 1-13. https://doi. org/10.1017/dmp.2020.103

Stambulova, N., Schinke, R. J., Lavallee, D., \& Wylleman, P. (2020). The COVID-19 pandemic and Olympic/ Paralympic athletes' developmental challenges and possibilities in times of a global crisis-transition. International Journal of Sport and Exercise Psychology. https://doi.org/10.1080/161219 7X.2020.1810865

Starzak, J., \& Sas-Nowosielski, K. (2019). Motivation of marathon runners in Poland. Polish Journal of Sport and Tourism, 26(4), 28-31. https://doi.org/10.2478/ pjst-2019-0023

Stoll, J., Müller, J. A., \& Trachsel, M. (2020). Ethical Issues in Online Psychotherapy: A Narrative Review. Frontiers in Psychiatry, 10, 993. https://doi.org/10.3389/ fpsyt.2019.00993

Sudano, L. E., Collins, G., \& Miles, C. M. (2016). Reducing barriers to mental health care for student-athletes: An integrated care model. Families, Systems and Health, 35(1), 77-84. https://doi.org/10.1037/ fsh0000242

Taku, K., \& Arai, H. (2020). Impact of COVID-19 on Athletes and Coaches, and Their Values in Japan: Repercussions of Postponing the Tokyo 2020 Olympic and Paralympic Games. Journal of Loss and Trauma, 25(8), 623-630. https://doi.org/10.1080/15325024.2 020.1777762

Tamir, I. (2020). Whatsappsport: Using Whatsapp While Viewing Sports Events. Journal of Sport and Social Issues, 44(3), 283-296. https://doi. org/10.1177/0193723520907624

Torres-Ramírez, M., García-Domingo, B., Aguilera, J., \& De La Casa, J. (2014). Video-sharing educational tool applied to the teaching in renewable energy subjects. Computers \& Education, 73, 160-177. https:// doi.org/10.1016/j.compedu.2013.12.014

da Silva, R. M., Rodrigues, C. J., De Medeiros, P. M., \& Rolim, M. L. (2021). Psychological effects caused by the COVID-19 pandemic in health professionals: A systematic review with meta-analysis. Progress in Neuro-Psychopharmacology and Biological Psychiatry, 104, 110062. https://doi.org/10.1016/j. pnpbp.2020.110062

Trujillo, D., \& Reyes-Bossio, M. (2019). Programa basado en mindfulness para la reducción de la ansiedad precompetitiva en deportistas de artes marciales. Retos, 36, 418-426. https://recyt.fecyt.es/index. php/retos/article/view/66589/43874

Urzúa, A., Vera-Villarroel, P., Caqueo-Urízar, A., \& Polanco-Carrasco, R. (2020). La Psicología en la prevención y manejo del COVID-19. Aportes desde la evidencia inicial. Terapia Psicologica, 38(1), 103-118. https://doi.org/10.4067/S0718-48082020000100103

Weinberg, R., \& Gould, D. (2010). Fundamentos de Psicología del Deporte y del Ejercicio Físico (4th ed.). Editorial Médica Panamericana.

Weinberg, R., Neff, R., \& Jurica, B. (2012). Online mental training: Making it available for the masses. Journal of Sport Psychology in Action, 3(3), 182-192. https://doi.org/10.1080/21520704.2012.656833

World Health Organization. (2020). Coronavirus disease 2019 ( COVID-19) : situation report, 29. https:// apps.who.int/iris/handle/10665/331118

Yang, H., Wen, X., \& Xu, F. (2020). The Influence of Positive Emotion and Sports Hope on Pre-competition State Anxiety in Martial Arts Players. Frontiers in Psychology, 11, 1460. https://doi.org/10.3389/ fpsyg.2020.01460

Yang, Y., Li, W., Zhang, Q., Zhang, L., Cheung, T., \& Xiang, Y. T. (2020). Mental health services for older adults in China during the COVID-19 outbreak. The Lancet Psychiatry, 7(4), e19. https://doi.org/10.1016/S22150366(20)30079-1

RIDU / Revista Digital de Investigación en Docencia Universitaria / e-ISSN: 2223-2516

(C) Los autores. Este artículo es publicado por la Revista Digital de Investigación en Docencia Universitaria del Área de Institutional Research and Effectiveness de la Dirección de Aseguramiento de la Calidad, Universidad Peruana de Ciencias Aplicadas. . "Este es un artículo de acceso abierto, distribuido bajo los términos de la LicenciaCreativeCommons Atribución 4.0 Internacional (CC BY 4.0) (https://creativecommons.org/licenses/by/4.0/deed.es), que permite el uso, distribución y reproducción en cualquier medio, siempre que la obra original sea debidamente citada." 